\title{
To be or not to be: the case of the hot WHIM absorption in the blazar PKS 2155-304 sight line
} \author{
P. Heinämäki ${ }^{9}$, E. Saar ${ }^{1}$, and A. Finoguenov ${ }^{3}$ \\ 1 Tartu Observatory, University of Tartu, 61602 Tõravere, Tartumaa, Estonia \\ e-mail: jukka@to.ee \\ 2 Leibniz-Institut für Astrophysik Potsdam (AIP), An der Sternwarte 16, 14482 Potsdam, Germany \\ 3 Department of Physics, University of Helsinki, Gustaf Hällströmin Katu 2a, 00014 Helsinki, Finland \\ ${ }^{4}$ University of Alabama in Huntsville, Huntsville, AL 35899, USA \\ 5 Department of Physics \& Astronomy, Regis University, Denver, CO 80221, USA \\ ${ }^{6}$ SRON Netherlands Institute for Space Research, Sorbonnelaan 2, 3584 CA Utrecht, The Netherlands \\ 7 Leiden Observatory, Leiden University, Niels Bohrweg 2, 2300 RA Leiden, The Netherlands \\ 8 Xiamen University, No. 422, Siming South Road, Xiamen, Fujian, PR China \\ 9 Tuorla Observatory, Väisäläntie 20, 21500 Piikkiö, Finland
}

J. Nevalainen ${ }^{1}$, E. Tempel ${ }^{1,2}$, J. Ahoranta ${ }^{3}$, L. J. Liivamägi ${ }^{1}$, M. Bonamente ${ }^{4}$, E. Tilton ${ }^{5}$, J. Kaastra ${ }^{6,7}$, T. Fang ${ }^{8}$,

Received 27 March 2018 / Accepted 22 November 2018

\begin{abstract}
The cosmological missing baryons at $z<1$ most likely hide in the hot $\left(T \gtrsim 10^{5.5} \mathrm{~K}\right)$ phase of the warm hot intergalactic medium (WHIM). While the hot WHIM is hard to detect due to its high ionisation level, the warm $\left(T \lesssim 10^{5.5} \mathrm{~K}\right)$ phase of the WHIM has been very robustly detected in the far-ultraviolet (FUV) band. We adopted the assumption that the hot and warm WHIM phases are co-located and therefore used the FUV-detected warm WHIM as a tracer for the cosmologically interesting hot WHIM. We performed an X-ray follow-up in the sight line of the blazar PKS 2155-304 at the redshifts where previous FUV measurements of $\mathrm{O}$ vI, Si IV, and broad Lyman-alpha (BLA) absorption have indicated the existence of the warm WHIM. We looked for the O vII He $\alpha$ and $\mathrm{O}$ viII Ly $\alpha$ absorption lines, the most likely hot WHIM tracers. Despite the very large exposure time $(\approx 1 \mathrm{Ms})$, the Reflection Grating Spectrometer unit 1 (RGS1) on-board XMM-Newton data yielded no significant detection which corresponds to upper limits of $\log N\left(\mathrm{O}\right.$ vII $\left.\left(\mathrm{cm}^{-2}\right)\right) \leq 14.5-15.2$ and $\log N\left(\mathrm{O} \mathrm{vIII}\left(\mathrm{cm}^{-2}\right)\right) \leq 14.9-15.2$. An analysis of the data obtained with the combination of the Low Energy Transmission Grating (LETG) and the High Resolution Camera (HRC) on-board Chandra yielded consistent results. However, the data obtained with the LETG, combined with the Advanced CCD Imaging Spectrometer (ACIS) lead to the detection of an feature resembling an absorption line at $\lambda \approx 20 \AA$ at simple one-parameter confidence level of $3.7 \sigma$, consistent with several earlier LETG/ACIS reports. Given the high statistical quality of the RGS1 data, the possibility of RGS1 accidentally missing the true line at $\lambda \sim 20 \AA$ is very low: $0.006 \%$. Neglecting this, the LETG/ACIS detection can be interpreted as Ly $\alpha$ transition of O vIII at one of the redshifts $(z \approx 0.054)$ of FUV-detected warm WHIM. Given the very convincing X-ray spectral evidence for and against the existence of the $\lambda \sim 20 \AA$ feature, we cannot conclude whether or not it is a true astrophysical absorption line. Considering cosmological simulations, the probability of the LETG/ACIS $\lambda \sim 20 \AA$ feature being due to the astrophysical O vIII absorber co-located with the FUV-detected O VI absorber is at the very low level of $\lesssim 0.1 \%$. We cannot completely rule out the very unlikely possibility that the LETG/ACIS $20 \AA$ feature is due to a transient event located close to the blazar.
\end{abstract}

Key words. BL Lacertae objects: individual: PKS 2155-304 - intergalactic medium - large-scale structure of Universe cosmology: observations

\section{Introduction}

High-resolution X-ray spectroscopy is currently a popular method for searching for the local $(z<1)$ missing baryons. According to large-scale cosmological simulations (e.g. Cen \& Ostriker 1999; Davé et al. 2001; Dolag et al. 2006; Branchini et al. 2009; Cui et al. 2012, 2018) these missing baryons reside in the hottest $\left(T \gtrsim 10^{5.5} \mathrm{~K}\right)$ phase of the warm hot intergalactic medium (WHIM), embedded within the filaments of the cosmic web. Given a bright enough background X-ray emission source, and a high enough column density of the intervening hot WHIM filament, detectable high ion metal absorption line features (e.g. $\mathrm{O}$ viI $\mathrm{He} \alpha$ and $\mathrm{O}$ vIII $\mathrm{Ly} \alpha$ ) can be imprinted in the emission spectrum.
Due to the relatively weak X-ray signal of the WHIM and the limitations of the sensitivities of the current instrumentation, significant measurements (at statistical significance $>3 \sigma$ ) of absorption lines from the ionised metals in the WHIM embedded within the large-scale filaments are very sparse. The rare 4- $\sigma$ detection of the $\mathrm{O}$ vII $\mathrm{He} \alpha$ line at the Sculptor Wall (Buote et al. 2009; Fang et al. 2010) has been called into question by 1) the existence of a galaxy $240 \mathrm{kpc}$ away from the absorber (Williams et al. 2013) and 2) the possible contamination by the Galactic $\mathrm{O}_{\text {II }} \mathrm{K} \beta$ absorption (Nicastro et al. 2016) whose rest wavelength, $\lambda \approx 22.30 \AA$, matches that of $\mathrm{O}$ vII $\mathrm{He} \alpha$ at $z=0.03$, as in the case of the Sculptor Wall. We showed in Nevalainen et al. (2015) that an unrealistically long path length through the halo of the nearby galaxy is required for the hydrogen column density inferred from the X-ray measurement in 
Sculptor Wall. Also, the X-ray measurements of the Galactic $\mathrm{O}$ II $\mathrm{K} \beta$ are questionable since the modelling heavily relies on the $\mathrm{O}_{\text {II }} \mathrm{K} \alpha$ line whose wavelength, $\lambda \approx 23.35 \AA$, coincides with the poorly calibrated instrumental feature in the spectrometers Reflection Grating Spectrometer unit 1 (RGS1) on-board XMM-Newton and the Low Energy Transmission Grating (LETG) on-board Chandra, resulting in significantly different equivalent widths depending on which instrument is used (see Nevalainen et al. 2017, and the references therein). Furthermore, the spectral modelling in Nicastro et al. (2016), if assumed to be correct, does not rule out a significant amount of $\mathrm{O}$ VII $\mathrm{He} \alpha$ absorption at $z=0.03\left(\log N\left(\mathrm{O}\right.\right.$ VII $\left.\left.\left(\mathrm{cm}^{-2}\right)\right)=15.4\right)$.

Nicastro et al. (2018) reported on the search for the WHIM absorption line in the sight line towards a blazar 1ES $1553+113$. When considering the redshift trials for the blind search and the systematic uncertainties of the instrument, they determined an absorption-line-like feature at $\lambda \sim 30.98 \AA$ at a 3.5- $\sigma$ confidence level and interpreted it as being due to $\mathrm{O}$ viI $\mathrm{He} \alpha$ at $z \sim 0.43$. It is not clear whether the signal comes from WHIM located in a large-scale filament (as required for the solution for the cosmological missing baryons problem) or from the halo of a nearby galaxy (as the authors prefer). Therefore, the interpretation of the signal as being due to $\mathrm{O}$ vII within a large-scale filament needs to be confirmed by detection of a galaxy filament using sufficiently deep spectroscopic data. The significance of the second line interpreted as $\mathrm{O}$ vII at $z \sim 0.36$ discussed in Nicastro et al. (2018) has a significance below the $3-\sigma$ limit.

On the other hand, the commonly used background sources, the blazars, are much brighter in the far-ultraviolet (FUV) compared to X-rays. Also, the current and past major FUV instruments, that is, the Cosmic Origins Spectrograph (COS) and the Space Telescope Imaging Spectrograph (STIS) on-board the Hubble Space Telescope (HST) and the ones on-board the Far Ultraviolet Spectroscopic Explorer (FUSE), are more sensitive than the current high-resolution X-ray instruments (RGS and LETG). Thus, the FUV measurements have yielded numerous detections of the extragalactic O VI and broad Ly $\alpha$ (BLA) absorption lines, typically interpreted as signatures of the warm $\left(T \lesssim 10^{5.5} \mathrm{~K}\right.$ ) WHIM (e.g. Lehner et al. 2007). The warm part of the local WHIM is therefore considered to be robustly detected (Shull et al. 2012).

Given the abundance of the FUV absorbers, it is tempting to use their locations to look for the hottest WHIM. The underlying assumption of the colocation of the warm (O vI and BLA) and hot (O VII-VIII) WHIM absorbers has not yet been well tested, basically due to the very limited number of significant $\mathrm{O}$ VII-VIII detections. We adopted the above assumption, performing an Xray follow-up in the sight line of the blazar PKS 2155-304 at the redshifts where previous FUV measurements of O VI, Si IV, and BLA absorption have indicated the existence of the warm WHIM.

Yao et al. (2009) stacked the available LETG/ACIS and MEG/ACIS data of PKS 2155-304 together with the spectra from several other AGN sight lines in order to examine the combined signal of the possible hot counterparts to the FUVdetected $\mathrm{O}$ vi absorbers. They obtained no significant absorption line and upper limits of $\log N\left(\mathrm{O}\right.$ vII $\left.\left(\mathrm{cm}^{-2}\right)\right) \leq 14.6$ and $\log N\left(\mathrm{O}\right.$ viII $\left.\left(\mathrm{cm}^{-2}\right)\right) \leq 15.5$. Compared to Yao et al. (2009) we have the luxury of a very large amount of high-resolution X-ray data on PKS 2155-304. We used all useful data available to us on PKS 2155-304 obtained with RGS1 (the RGS2 unit does not cover most of the studied lines) and LETG/ACIS and LETG/HRC-S combinations (the quality of the available Medium Energy Grating (MEG) on-board Chandra data was too poor to yield meaningful constraints). We use these data in order to obtain similar detection limits as Yao et al. (2009) but for the individual FUV absorbers in a single sight line, that is, towards PKS 2155-304, as reported by Tilton et al. (2012). While doing so, we avoid possible problems due to stacking the data from different instruments, targets, and redshifts. With this we aim at improving the observational status of the possible colocation of the warm and hot WHIM.

We use $\Omega_{\mathrm{m}}=0.3, \Omega_{\Lambda}=0.7$ and $H_{0}=70 \mathrm{~km} \mathrm{~s}^{-1} \mathrm{Mpc}^{-1}$. The distances and redshifts refer to the heliocentric frame. We quote uncertainties at the $1 \sigma$ confidence level.

\section{FUV detections}

In the present work we employ the catalogue of blazar FUV measurements from Tilton et al. (2012). The PKS 2155304 sight line has been extensively studied using COS (Savage et al. 2014, Danforth et al. 2016) as well as with STIS and FUSE (e.g. Shull et al. 1998, 2003; Sembach et al. 2003; Wakker et al. 2003; Danforth \& Shull 2008; Stocke et al. 2013, 2014; Richter et al. 2017). The inclusion of the FUSE and the STIS instruments ensures the coverage of $\mathrm{O}$ vi lines at all redshifts of interest due to their broader wavelength range compared to the COS.

The BLA and O vi absorption, observable in the FUV band, are commonly interpreted as originating from the warm WHIM. Using a single O vi transition of the 1031.9/1037.6 doublet or a single BLA as a WHIM signature may be too optimistic, given the possibilities of misidentification of a single line. On the other hand, a more robust criterion of requiring both $\mathrm{O}_{\mathrm{VI}}$ transitions to be significantly detected reduces the number of the potential WHIM redshifts due to, for example, the detector gap at the critical wavelengths, and consequently we may loose some true WHIM signal. We therefore adopted the more relaxed criterion of requiring at least one significantly $(3 \sigma)$ detected $\mathrm{O}_{\mathrm{VI}}$ line (or other metal line whose ionisation temperature exceeds $10^{5} \mathrm{~K}$ ) or BLA for follow-up, keeping the above caveat in mind.

As BLA we considered absorption lines due to $\mathrm{H}_{\mathrm{I}} \mathrm{Ly} \alpha$ transition with broadening velocity higher than $40 \mathrm{~km} \mathrm{~s}^{-1}$, which corresponds to thermal broadening of hydrogen at $T=10^{5} \mathrm{~K}$, the lower limit of the WHIM temperature range.

Using the above criteria, the catalogue of Tilton et al. (2012) yielded O vI, Si Iv, or BLA detections at nine different redshifts in the PKS 2155-304 sight line (see Table 1). Considering the LETG/RGS photon energy resolution of $40-60 \mathrm{~m} \AA$ at $\lambda \approx 20 \AA$, we associated the FUV absorbers with redshift differences smaller than 0.002 (co-moving distance difference of $\sim 8 \mathrm{Mpc}$ ) with a single X-ray absorber. For such absorbers, that is, A3, A4, and A6 we use the average redshift of the FUV lines as the X-ray follow-up redshift. As a result we have the redshifts of six possible X-ray absorbers to study with X-ray instruments in the PKS 2155-304 sight line (see Table 1).

\section{X-ray analysis}

Here we examine the most likely hot WHIM tracers, that is, O VII $\mathrm{He} \alpha$ and $\mathrm{O}$ VIII Ly $\alpha$ lines at the five FUV-predicted redshifts (see Table 1), including the much studied and controversial O viII Ly $\alpha$ line at $z=0.054-0.056$ (see Sect. 4.1). 
Table 1. FUV WHIM absorbers in the sight line to blazar PKS 2155-304.

\begin{tabular}{|c|c|c|c|c|c|c|c|c|c|c|}
\hline \multirow[t]{3}{*}{$\mathrm{ID}^{a}$} & \multirow[t]{3}{*}{$z_{\mathrm{FUV}}{ }^{b}$} & \multicolumn{6}{|c|}{ Line } & \multirow[t]{3}{*}{$z_{\mathrm{X}-\text {-ray }}{ }^{c}$} & \multirow{3}{*}{$\begin{array}{c}\lambda(\mathrm{O} \text { vII He } \alpha)^{d} \\
(\AA) \\
\end{array}$} & \multirow{3}{*}{$\begin{array}{c}\lambda(\mathrm{O} \text { viII Ly } \alpha)^{d} \\
(\AA)\end{array}$} \\
\hline & & \multicolumn{3}{|c|}{ Metal } & \multicolumn{3}{|c|}{ BLA } & & & \\
\hline & & Ion & $\begin{array}{l}\mathrm{EW}^{e} \\
(\mathrm{m \AA})\end{array}$ & $\log N\left(\mathrm{~cm}^{-2}\right)^{f}$ & $\begin{array}{l}\mathrm{EW}^{g} \\
(\mathrm{m \AA})\end{array}$ & $\begin{array}{c}b^{h} \\
\left(\mathrm{~km} \mathrm{~s}^{-1}\right)\end{array}$ & $\log N\left(\mathrm{~cm}^{-2}\right)^{i}$ & & & \\
\hline A1 & 0.00878 & - & - & & $49 \pm 8$ & $59 \pm 8$ & $13.0 \pm 0.1$ & 0.00878 & 21.79 & 19.14 \\
\hline $\mathrm{A} 2$ & 0.01892 & - & - & & $59 \pm 4$ & $38 \pm 4$ & $13.0 \pm 0.1$ & 0.01892 & 22.01 & 19.33 \\
\hline $\mathrm{A} 3 \mathrm{a}^{j}$ & 0.05405 & $\mathrm{O}_{\mathrm{VI}}$ & $32 \pm 5^{k}, 30 \pm 9^{k}$ & $13.6 \pm 0.1$ & $315 \pm 4$ & $44 \pm 0$ & $14.1 \pm 0.1$ & 0.05425 & 22.77 & 20.00 \\
\hline $\mathrm{A} 3 \mathrm{~b}^{j}$ & 0.05445 & - & - & & $60 \pm 31$ & $54 \pm 7$ & $13.0 \pm 0.1$ & & & \\
\hline $\mathrm{A} 4 \mathrm{a}^{j}$ & 0.05659 & - & - & & $477 \pm 10$ & $48 \pm 1$ & $14.5 \pm 0.3$ & 0.05683 & 22.83 & 20.05 \\
\hline $\mathrm{A} 4 \mathrm{~b}^{j}$ & 0.05707 & $\mathrm{O}_{\mathrm{VI}}$ & $44 \pm 11^{l}$ & $13.6 \pm 0.1$ & $424 \pm 11$ & $68 \pm 1$ & $14.0 \pm 0.0$ & & & \\
\hline A5 & 0.08062 & Si IV & $12 \pm 4^{m}$ & $12.1 \pm 0.1$ & $29 \pm 5$ & $40 \pm 5$ & $12.7 \pm 0.1$ & 0.08062 & $(23.34)^{n}$ & 20.50 \\
\hline $\mathrm{A} 6 \mathrm{a}^{j}$ & 0.10552 & - & - & & $360 \pm 7$ & $54 \pm 1$ & $14.1 \pm 0.2$ & 0.10569 & 23.83 & 20.97 \\
\hline $\mathrm{A} 6 \mathrm{~b}^{j}$ & 0.10586 & - & - & & $156 \pm 22$ & $66 \pm 4$ & $13.5 \pm 0.0$ & & & \\
\hline
\end{tabular}

Notes. ${ }^{(a)}$ Name of absorber. ${ }^{(b)}$ Redshift of FUV absorber. ${ }^{(c)}$ Our adopted redshift for the X-ray follow-up. ${ }^{(d)}$ Redshifted wavelength of O viI He $\alpha$ and $\mathrm{O}$ vIII Ly $\alpha .{ }^{(e)}$ Equivalent width of the metal line. ${ }^{(f)}$ Column density of the metal line. ${ }^{(g)}$ Equivalent width of the BLA. ${ }^{(h)}$ Doppler parameter of the BLA. ${ }^{(i)}$ Column density of the BLA. ${ }^{(j)}$ Two FUV absorbers at the same WHIM structure candidate are shown separately. ${ }^{(k)}$ Equivalent width of the O vi $1031.9 \AA$ and $1037.6 \AA$ transitions, respectively. ${ }^{(l)}$ Equivalent width of the only significantly detected O vi $1031.9 \AA$ transition. ${ }^{(m)}$ Equivalent width of the only significantly detected Si Iv $1393.8 \AA$ transition. ${ }^{(n)}$ The possible A5 O viII line would land at problematic wavelengths due to astrophysical and instrumental oxygen edges which prevents an accurate modelling; we therefore excluded this line from further analyses.

\subsection{X-ray data}

We analysed all PKS 2155-304 spectra obtained with RGS1 and LETG/HRC-S available in the year 2016, published in Nevalainen et al. (2017). The RGS2 unit does not cover most of the interesting wavelengths and we therefore ignore that data. We examine LETG/ACIS spectra separately in Sect. 4.4. The exposure time of the RGS1 exceeds $1 \mathrm{Ms}$, rendering the data very powerful for measuring the weak WHIM lines.

We analysed the data using the SPEX spectral fitting package (Kaastra et al. 1996). We used a bin size of $20 \mathrm{~m} \AA$ for RGS and $25 \mathrm{~m} \AA$ for LETG/HRC, oversampling the spectral resolution by a factor of 2-3. Due to the high number of counts in the spectral channels, we used $\chi^{2}$ statistics. We added $2 \%$ of the flux at each channel as a systematic uncertainty of the effective area in quadrature to the statistical uncertainties; see Nevalainen et al. (2017).

\subsection{Wavelength scale calibration}

To correct for possible wavelength scale calibration offsets, the individual RGS spectra have been shifted as indicated by the Galactic neutral absorber lines before co-addition (see the description and references in Nevalainen et al. 2017).

The same procedure was not practical in the case of HRC spectra, since its lower effective area and exposure time ( $\sim 300 \mathrm{ks})$ compared to RGS rendered the statistical quality too poor for measuring the Galactic line centroids accurately using the individual spectra. We therefore examined the total HRC spectrum, co-added without any shifts. The Galactic O I $(\lambda=$ $23.51 \AA)$ and $\mathrm{O}_{\text {VII }}(\lambda=21.60 \AA)$ lines were unambiguously identified. We fitted the 23.0-24.0 $\AA$ and 21.0-22.0 $\AA$ bands with a model consisting of a power-law continuum and a Gaussian line and consequently obtained significant detections of the Galactic $\mathrm{O}_{\mathrm{I}}$ and $\mathrm{O}$ vII lines. Their centroid wavelengths were detected with an accuracy better than $\pm 10 \mathrm{~m} \AA$. Within the uncertainties, the centroid wavelengths agreed with the a priori values. The HRC energy scale of the co-added spectrum was therefore very accurately calibrated.

\subsection{Wavebands}

Our aim is to cover the possible $\mathrm{O}$ vII He $\alpha$ and $\mathrm{O}$ vIII Ly $\alpha$ lines, assuming the redshifts of the absorbers A1-A6 (see Table 1 for the wavelengths). We wish to select minimally wide bands around the above lines to simplify the continuum modelling, but wide enough to obtain a robust continuum level. We decided to confine the analyses within wavebands $21.7-24.0 \AA$ (the O VII band) and 19.05-21.1 $\AA$ (the O viII band).

We excluded some problematic channels, as described in the following. In both RGS1 and HRC the wavelength of the A5 O vII absorber $(\lambda \approx 23.34 \AA)$ coincides with the instrumental absorption feature (de Vries et al. 2003, 2015) rendering the measurement inaccurate. We thus excluded the 22.85-23.6 band from both spectra and consequently did not analyse the possible A5 O viI. Due to the RGS1 CCD4-5 gap we excluded the 20.75-20.90 $\AA$ band from the RGS1 analysis. Fortunately, none of our candidate lines has the centroid in that band. There is excess of HRC data on top the otherwise smooth continuum at 19.74-19.85 $\AA$ which cannot be adequately fitted. We therefore excluded the HRC data in this band which does not contain any of the tested lines.

\subsection{Galactic absorption}

For the ionised Galactic absorption, we adopted the Nevalainen et al. (2017) model: the hot halo (HH) and the transition temperature gas (TTG), manifested as O Iv, O v, O vII, $\mathrm{O}$ vIII, N vi, C VI and Ne IX absorption lines, were modelled as two CIE absorbers (a model called "hot" in SPEX). We allowed the parameters of these components to vary within the statistical 1- $\sigma$ uncertainties as derived from RGS1 data in Nevalainen et al. (2017): $N\left(H_{\mathrm{HH}}\right)=2.4 \pm 0.3 \times 10^{19} \mathrm{~cm}^{-2}$, $k T_{\mathrm{HH}}=1.7 \pm 0.1 \times 10^{-1} \mathrm{keV}, N\left(H_{\mathrm{TTG}}\right)=1.0 \pm 0.2 \times 10^{19} \mathrm{~cm}^{-2}$, $k T_{\text {TTG }}=1.4 \pm 0.1 \times 10^{-2} \mathrm{keV}$. We assumed the element number density ratios from Lodders \& Palme (2009) and that the metal abundance is solar. We constrained the non-thermal broadening 
velocity to $15-35 \mathrm{~km} \mathrm{~s}^{-1}$, as in Nevalainen et al. (2017) based on the FUV measurements of Wakker (priv. comm.).

For the LETG/HRC, we additionally included the neutral Galactic disc absorber consisting of atomic and molecular components which have been used to correct for the cold Galactic absorption when processing the RGS spectra (see Nevalainen et al. 2017 for details).

The band passes occupied by the possible O viI line due to A3 and A4 overlap with that of the Galactic O Iv line (see Nevalainen et al. 2017 for the discussion of the identification of the line as Galactic O IV). However, since we used priors in the Galactic absorber model (see above), the fit converged to a $\chi^{2}$ minimum.

\subsection{Blazar emission}

The RGS spectra have already been normalised to the PKS 2155-304 continuum, that is, the PKS 2155-304 emission absorbed by the cold Galactic component; see Nevalainen et al. (2017). Therefore, here we modelled the RGS blazar emission with a constant, which we allowed to vary in order to accommodate for the statistical uncertainties of the continuum modelling.

The emission spectrum of PKS 2155-304 as measured with HRC was adequately modelled in the $\mathrm{O}$ VII band with a power law. In the WHIM analysis below, we allowed the normalisation and the photon index of the power-law component to vary. However, in the case of the HRC O vIII band the continuum was more complicated. We modelled that with a spline model with a constant grid size of $300 \mathrm{~m} \AA$. Allowing all the spline parameters to be free in the WHIM analysis allowed too much freedom to the model in the sense that some of the tested lines were significantly affected. We therefore fixed the spline parameters to their best fit values in order to maintain the continuum shape and allowed only the normalisation of the continuum to vary.

\subsection{WHIM lines}

In order to examine the possible WHIM lines, we added a SPEX model called slab to the continuum model described above. "Slab" calculates the transmission of a thin slab of material whose ion column densities can be varied independently, that is, the ion ratios are not determined by the ionisation balance. The Lorentz component of the final Voigt profile is calculated for each transition in the SPEX atomic data base for a given ion, while the Gaussian component is calculated based on the input value of the total velocity dispersion (thermal and non-thermal). We fixed the total broadening to $100 \mathrm{~km} \mathrm{~s}^{-1}$, which corresponds to the pure thermal broadening of oxygen at $T=10^{7} \mathrm{~K}$. Thus, in the case of significant non-thermal broadening, our measurements of the column densities of $\mathrm{O}_{\text {VII }}$ and $\mathrm{O}$ VIII are somewhat overestimated. We get back to this point when discussing the measurements below.

We fitted the data of each instrument separately, fixing the slab model wavelength to that of either $\mathrm{O}$ vII $\mathrm{He} \alpha$ or O vIII Ly $\alpha$, applying one of the adopted X-ray redshifts at a time for a given fit (see Table 1). The column density of a given ion was the only free parameter.

We obtained the best-fit values and the uncertainties (including both the statistical uncertainty and the systematic $2 \%$ uncertainty on the flux) of the column densities by the $\chi^{2}$ minimisation. We used these models to calculate the equivalent width, and its uncertainties $\left(\mathrm{EW} \pm \sigma_{\mathrm{EW}}\right)$, of each tested line and used the ratio $\mathrm{EW} / \sigma_{\mathrm{EW}}$ as a measure of its detection significance.

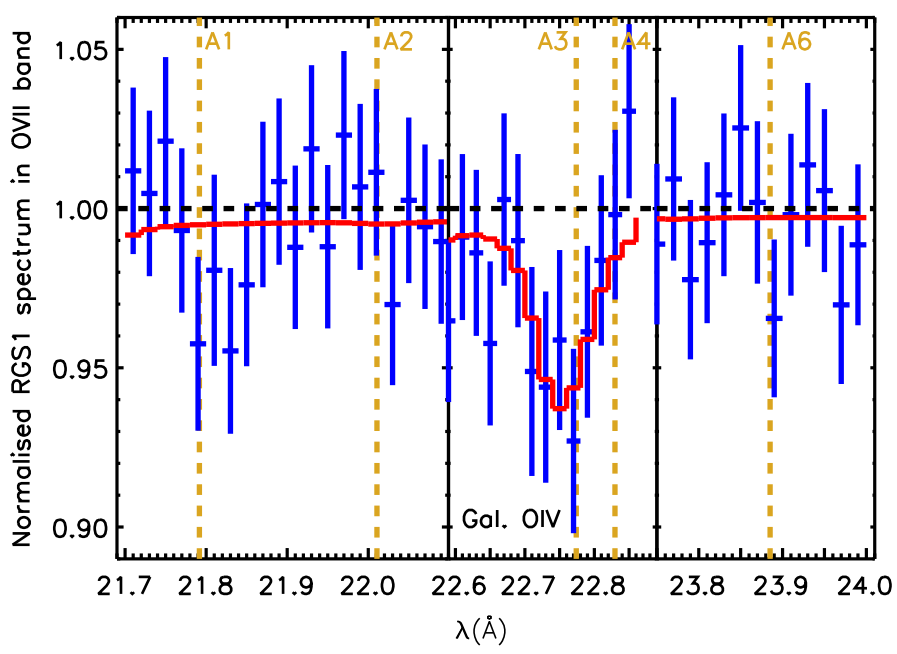

Fig. 1. Normalised RGS1 data (blue crosses) and the normalised bestfit model (red line) for the PKS 2155-304 emission absorbed with the Galactic neutral disk, hot halo and the transition temperature gas TTG (see Nevalainen et al. 2017). The normalisation is done by dividing the spectra by the best-fit model consisting of the PKS 2155-304 emission and only the Galactic neutral absorber to highlight the effect of the Galactic halo and TTG. In particular, the Galactic O Iv absorption line overlaps with the $\mathrm{O}$ vir test line for A3 (middle panel). The dashed vertical lines indicate the centroid wavelengths of the $\mathrm{O}$ VII test lines assuming the redshifts of the structures A1, A2, A3, A4 and A6 (A5 is missing due to the overlap of its centroid wavelength with the instrumental feature in both RGS1 and LETG; see de Vries et al. 2003, 2015). The error bars contain both the statistical uncertainties and the systematic one ( $2 \%$ of the flux).

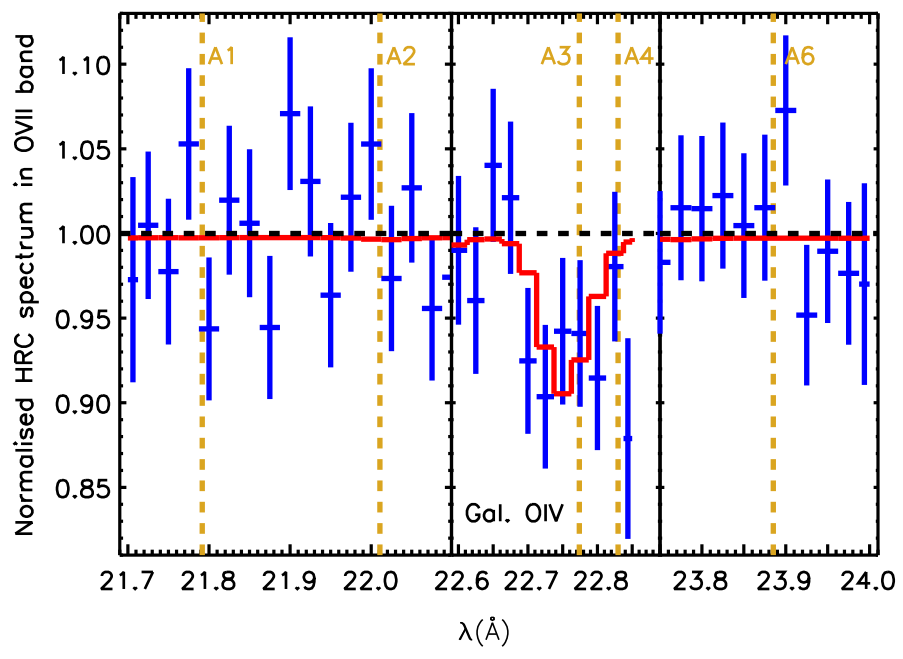

Fig. 2. As in Fig. 1 but for the LETG/HRC instrument combination.

\subsection{Results}

We found that none of the tested lines were detected significantly with RGS1 or HRC (see Figs. 1-4 and Table 2). The upper limits ${ }^{1}$ of a few milliangstrom correspond to $\mathrm{O}$ VII and $\mathrm{O}$ VIII levels of $\sim 10^{15} \mathrm{~cm}^{-2}$. We discuss the implications of these results in Sect. 5.

The underlying assumption in the above analysis is that the warm and hot WHIM are co-located and at rest (or moving with

\footnotetext{
1 The obtained upper limits for the column densities may be slightly overestimated due to the possible non-thermal velocities which we neglected.
} 


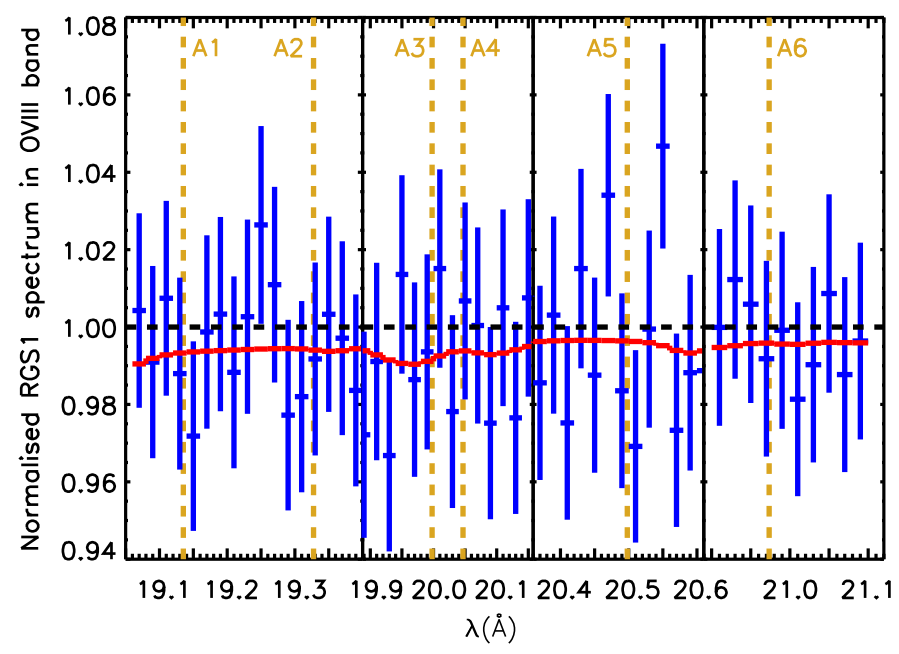

Fig. 3. As in Fig. 1 but for the $\mathrm{O}$ VIII line.

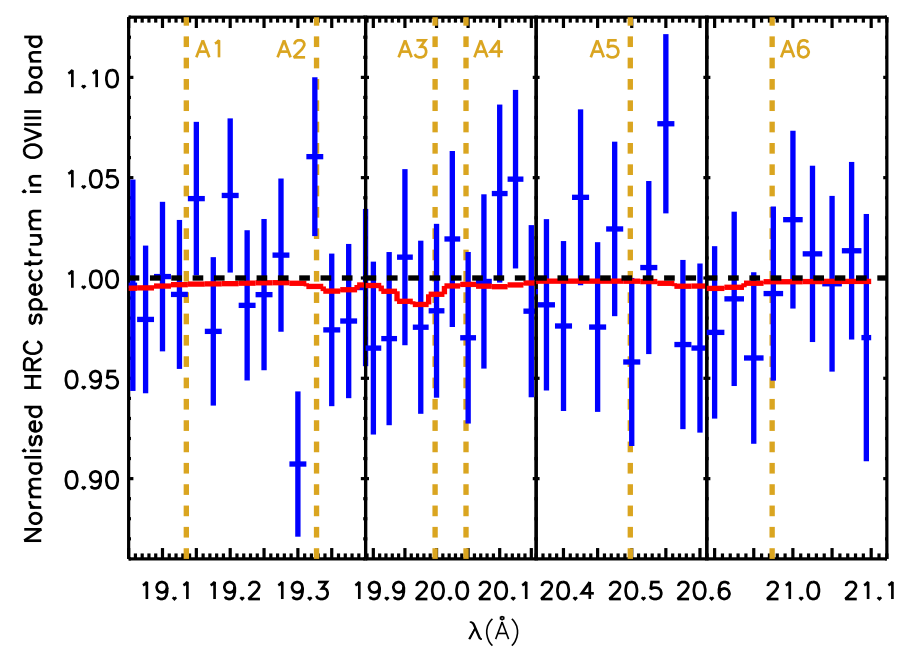

Fig. 4. As in Fig. 2 but for the $\mathrm{O}$ vIII line.

the same velocity), so that $z_{\mathrm{X} \text {-ray }}=z_{\mathrm{FUV}}$. We next relaxed this assumption by allowing a relative projected sight line velocity difference up to $\pm 600 \mathrm{~km} \mathrm{~s}^{-1}$, that is, $\Delta_{Z}= \pm 0.002$ ) between the FUV and X-ray absorbers, while co-located. In practise, we repeated the above fits, but shifting the test line centroid by $\pm 40 \mathrm{~m} \AA$ in steps of $20 \mathrm{~m} \AA$. This resulted in no significant change in the results.

\section{4. $\mathrm{O}$ vil Ly $\alpha$ absorption at $z \sim 0.054$ ?}

Before discussing the implications of the above results, we investigate here the widely reported line-like feature at $\lambda \sim 20 \AA$ in the LETG/ACIS PKS 2155-304 spectrum, interpreted as O vIII Ly $\alpha$ absorption at $z=0.054-0.056$ (see Table 3 ). The redshift of this line agrees with the range of values of the FUV lines associated with our A3 absorber (see Table 1). We use a notation $\mathrm{O}$ VIII $\mathrm{A} 3$ for this line in the following.

\subsection{Summary of earlier $X$-ray work}

Combining three observations with a total exposure time of $80 \mathrm{ks}$ obtained with LETG/ACIS-S, Fang et al. (2002) reported a 4.5$\sigma$ detection of an absorption line at $\lambda=20.02 \pm 0.02 \AA$ and
Table 2. Equivalent widths and column densities for the hot WHIM.

\begin{tabular}{|c|c|c|c|c|}
\hline \multirow[t]{2}{*}{ Instr. } & \multicolumn{2}{|c|}{$\mathrm{O}$ viI $\mathrm{He} \alpha$} & \multicolumn{2}{|c|}{ O viII Ly $\alpha$} \\
\hline & $\begin{array}{c}\mathrm{EW}^{a} \\
\mathrm{~mA}\end{array}$ & $\begin{array}{c}\log N(\text { ion })^{a} \\
\mathrm{~cm}^{-2}\end{array}$ & $\begin{array}{c}\mathrm{EW}^{a} \\
\mathrm{m \AA}\end{array}$ & $\begin{array}{c}\log N(\text { ion })^{a} \\
\mathrm{~cm}^{-2}\end{array}$ \\
\hline \multicolumn{5}{|c|}{$\mathrm{A} 1\left(z=0.00878 ; \lambda_{\mathrm{O}_{\mathrm{VII}}}=21.79 \AA ; \lambda_{\mathrm{O} \text { IIII }}=19.14 \AA\right)$} \\
\hline RGS1 & $2.2 \pm 1.8$ & $14.9_{-0.8}^{+0.3}$ & $<2.1$ & $<15.2$ \\
\hline $\mathrm{HRC}$ & $<2.6$ & $<15.0$ & $<1.2$ & $<15.0$ \\
\hline \multicolumn{5}{|c|}{$\mathrm{A} 2\left(z=0.01892 ; \lambda_{\mathrm{O} \text { VII }}=22.01 \AA ; \lambda_{\mathrm{O} \text { VIII }}=19.33 \AA\right)$} \\
\hline RGS1 & $<1.2$ & $<14.6$ & $<2.0$ & $<15.2$ \\
\hline HRC & $<1.0$ & $<14.6$ & $<2.4$ & $<15.3$ \\
\hline \multicolumn{5}{|c|}{$\mathrm{A} 3\left(z=0.05425 ; \lambda_{\mathrm{O} \text { VII }}=22.77 \AA ; \lambda_{\mathrm{O} \text { III }}=20.00 \AA\right)$} \\
\hline RGS1 & $<4.0$ & $<15.2$ & $<1.0$ & $<14.9$ \\
\hline $\mathrm{HRC}$ & $<4.6$ & $<15.2$ & $<2.3$ & $<15.2$ \\
\hline \multicolumn{5}{|c|}{$\mathrm{A} 4\left(z=0.05683 ; \lambda_{\mathrm{O} \text { VII }}=22.83 \AA ; \lambda_{\mathrm{O} \text { III }}=20.05 \AA\right)$} \\
\hline RGS1 & $<0.8$ & $<14.5$ & $<1.4$ & $<15.0$ \\
\hline $\mathrm{HRC}$ & $<5.8$ & $<15.4$ & $<2.3$ & $<15.3$ \\
\hline \multicolumn{5}{|c|}{$\mathrm{A} 5\left(z=0.08062 ; \lambda_{\mathrm{O} \text { VII }}=23.34 \AA ; \lambda_{\mathrm{O} \text { III }}=20.50 \AA\right)$} \\
\hline RGS1 & $-{ }^{b}$ & $-b$ & $<1.9$ & $<15.2$ \\
\hline HRC & $-{ }^{b}$ & $-^{b}$ & $<2.6$ & $<15.3$ \\
\hline \multicolumn{5}{|c|}{ A6 $\left(z=0.10569 ; \lambda_{\mathrm{O}_{\mathrm{VII}}}=23.89 \AA ; \lambda_{\mathrm{O} \mathrm{VIII}}=20.97 \AA\right)$} \\
\hline RGS1 & $<1.8$ & $<14.8$ & $<1.4$ & $<15.0$ \\
\hline HRC & $<1.6$ & $<14.8$ & $<2.6$ & $<15.3$ \\
\hline
\end{tabular}

Notes. ${ }^{(a)}$ The constrains or the upper limits at $1 \sigma$ confidence level for the equivalent width (EW) and the ion column densities (log $N$ (ion)), where the uncertainties include both the statistical uncertainty and the systematic $2 \%$ uncertainty of the flux. ${ }^{(b)}$ The wavelengths coincide with the instrumental feature.

interpreted that as $\mathrm{O}$ vIII $\operatorname{Ly} \alpha$ absorption at $z=0.056 \pm 0.001$ (see Table 3 for the summary of different measurements). This was contrasted by Cagnoni et al. (2004) who studied $110 \mathrm{ks}$ of RGS1 data of PKS 2155-304 and did not detect the line despite the longer exposure and higher effective area. An another analysis with LETG/ACIS data (Fang et al. 2007), this time with a larger exposure time of $280 \mathrm{ks}$, compared to that in Fang et al. (2002), yielded a 5.0 $\sigma$ detection of an absorption line at $\lambda=$ $20.00 \pm 0.01 \AA$, consistent with the O VIII Ly $\alpha$ line at $z=$ $0.054 \pm 0.001$. The equivalent width of the line is consistent with that of Fang et al. (2002) within the uncertainties at $90 \%$ confidence level. Williams et al. (2007) analysed a LETG/ACIS data set, largely overlapping with Fang et al. (2007), and detected an absorption line at 3.5- $\sigma$ confidence level at $\lambda=20.03 \pm 0.01 \AA$, that is, at a slightly higher wavelength $(2 \sigma)$ than Fang et al. (2007), corresponding to $z=0.056 \pm 0.001$ if interpreted as the $\mathrm{O}$ vIII Ly $\alpha$ line. The equivalent widths agree very well in the two works. They also analysed $230 \mathrm{ks}$ of LETG/HRC data, which however did not yield a significant detection.

\subsection{Our results}

We used our RGS1 non-detection of $\mathrm{OVIII}_{\mathrm{A} 3}$, that is, $\mathrm{EW}\left(\mathrm{O} \mathrm{VIII}_{\mathrm{A} 3}\right) \leq 1.3 \mathrm{m \AA}$ (Table 2$)$, to estimate the upper limit for the column density as $\log N\left(\mathrm{O}_{\text {VIII }_{\mathrm{A} 3}}\left(\mathrm{~cm}^{-2}\right)\right) \leq 14.9$. This is by a factor of four smaller than the lower limit reported by Fang et al. (2007) and Williams et al. (2007) obtained with LETG/ACIS (Table 3). Also our LETG/HRC analysis yielded only an upper limit of $\mathrm{EW}\left(\mathrm{O} \mathrm{VIII}_{\mathrm{A} 3}\right) \leq 2.1 \mathrm{m \AA}$, significantly lower than the reported LETG/ACIS detections (see Fig. 5 and Table 3). 
Table 3. O vIII Ly $\alpha$ measurements at $z \sim 0.054-0.056$ in the PKS 2155-304 sight line.

\begin{tabular}{|c|c|c|c|c|c|c|c|c|}
\hline \multirow[t]{2}{*}{ Ref. } & \multicolumn{2}{|c|}{ RGS1 } & \multicolumn{2}{|c|}{ LETG/HRC } & \multicolumn{4}{|c|}{ LETG/ACIS-S } \\
\hline & $\begin{array}{l}\text { Exp. time } \\
(\mathrm{ks})\end{array}$ & $\begin{array}{l}\mathrm{EW}^{a} \\
(\mathrm{~mA})\end{array}$ & $\begin{array}{l}\text { Exp. time } \\
(\mathrm{ks})\end{array}$ & $\begin{array}{l}\mathrm{EW}^{a} \\
(\mathrm{~m} \AA)\end{array}$ & $\begin{array}{l}\text { Exp. time } \\
(\mathrm{ks})\end{array}$ & $z$ & $\begin{array}{l}\mathrm{EW}^{a} \\
(\mathrm{~m} \AA)\end{array}$ & Signif. $b$ \\
\hline $\mathrm{F} 02$ & - & - & - & - & 80 & $0.056 \pm 0.001$ & $14.0_{-34}^{+4.4}$ & $4.5 \sigma$ \\
\hline C04 & 110 & $\leq 7$ & - & - & - & - & -3.4 & - \\
\hline F07 & - & - & 200 & $\leq 5$ & 280 & $0.054 \pm 0.001$ & $7.4_{-12}^{+1.7}$ & $5.0 \sigma$ \\
\hline W07 & - & - & 230 & $\leq 6$ & 250 & $0.056 \pm 0.001$ & $7.5 \pm 2.1$ & $3.5 \sigma$ \\
\hline This work & 1200 & $\leq 1.3$ & 310 & $\leq 2.1$ & 330 & $0.0554 \pm 0.0004$ & $6.2 \pm 1.7$ & $3.2 \sigma$ \\
\hline
\end{tabular}

Notes. ${ }^{(a)}$ The equivalent width is reported at $1 \sigma$ confidence level. ${ }^{(b)}$ The significance of the detection.

References. F02: Fang et al. (2002). C04: Cagnoni et al. (2004). F07: Fang et al. (2007). W07: Williams et al. (2007). This work: the final sample.

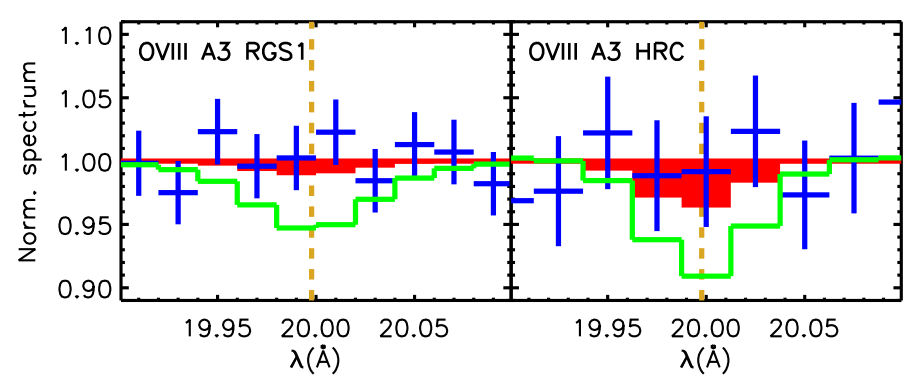

Fig. 5. Normalised data (blue crosses) and the $\mathrm{O}$ viII line models at the upper 1- $\sigma$ limit (red areas) at the redshift of A3 for RGS1 (left panel) and LETG/HRC-S (right panel). The normalisation is done by dividing the spectra by the best-fit model consisting of the PKS 2155-304 emission and all (neutral + ionised) Galactic absorbers. The vertical dashed lines indicate the centroid wavelength of the $\mathrm{O}$ viII doublet redshifted to $z=0.0544$. The green lines indicate the lower limit reported by Fang et al. (2007). The error bars contain both the statistical uncertainties and the systematic one ( $2 \%$ of the flux).

We investigate in the following what might be causing such large discrepancies.

\subsection{RGS issues}

As discussed in Sect. 4.1, the previous XMM-Newton/RGS work of a subsample of our data (Cagnoni et al. 2004) yielded no detection of $\mathrm{O}_{\mathrm{VIII}} \mathrm{A}$, while the upper limit for the equivalent width was inconsistent with the LETG/ACIS detections of Fang et al. (2007) and Williams et al. (2007). Our RGS analysis contains a lot more data and differs in several ways from the above RGS work (Cagnoni et al. 2004). Nonetheless, the results are consistent in the sense that neither analysis detected the $\mathrm{O}_{\mathrm{VIII}_{\mathrm{A} 3}}$ line, indicating no significant systematic uncertainties in either RGS results.

Co-addition of a large number of spectra, 25 in our case, may still be problematic considering weak unresolved lines. In order to examine the robustness of our results we repeated our $\mathrm{O} \mathrm{VIII}_{\mathrm{A} 3}$ analysis, but this time instead of co-adding the 25 RGS1 residual spectra (see Nevalainen et al. 2017 for the information on the observation identification codes), we analysed them jointly. Here we started from the archival data and processed them with the standard procedures available in October 2017, differently from the procedure described in Sect. 3. We fitted the 19.05-20.2 $\AA$ continua with a power-law model, allowing the normalisations to vary independently from each other.
We adopted the galactic absorption model described in Sect. 3.4. We added a 'slab' component (see Sect. 3.6) for O vIII absorption redshifted by $z \equiv 0.055$, forcing the O vIII column density equal in all spectra. The result is $\mathrm{EW}\left(\mathrm{O}_{\mathrm{VIII}}\right) \leq 0.9 \mathrm{~m} \AA$, very similar to what we derived using the co-added RGS1 spectrum $\mathrm{EW}\left(\mathrm{O}_{\mathrm{VIII}}\right) \leq 1.3 \mathrm{m \AA}$. Therefore, we conclude that we have not missed a significant $\mathrm{O}$ VIII Ly $\alpha$ line at $z=0.055$ due to possible co-addition problems in the RGS1 data. The close similarity of the two results also demonstrates that the non-standard processing of the RGS data we adopted for our work (see Nevalainen et al. 2017 for details) does not produce significant problems.

\subsection{LETG/ACIS issues}

The fact that the LETG/ACIS is the only instrument combination that has yielded significant detections of $\mathrm{O}_{\text {VIII }} \mathrm{A}_{3}$ indicates significant systematic uncertainties in the LETG/ACIS data. We therefore investigated the hypothesis that the LETG/ACIS $\mathrm{O}_{\text {VIII }} 3$ detections are actually due to a line-like artifact in the LETG/ACIS data as analysed by Fang et al. (2007) and Williams et al. (2007).

\subsubsection{Aim point offset}

As discussed by Fang et al. (2007) and Williams et al. (2007), most of the observations these authors used were obtained with an aim point offset of $\Delta y=+1.5$ arcmin. Such a configuration places the tentative positive first-order $20.0 \AA$ line close to the boundary between nodes 2 and 3 in the S3 chip. The standard dithering of the spacecraft (16 arcsec peak-to-peak or $\sim 1 \AA$ ) will fill the gap with photons. Depending on the completeness of the procedure, the calibration of the effective area at $20.0 \AA$ may be less accurate than at other wavelengths.

Fang et al. (2007) presented the following arguments against significant calibration artifacts at $\lambda \sim 20.0 \AA$. Firstly, the tentative negative first-order $\lambda=20.0 \AA$ line, when using the above offsets, is safely outside the node boundary. Yet, according to Fang et al. (2007) the line is clearly visible in both positive and negative first-order data. Unfortunately they did not report the wavelengths or equivalent widths for these lines. Secondly, the $20.0 \AA$ line obtained with observation 3669 , which has been carried out with an exceptionally large offset $(\Delta y=+3.3 \mathrm{arcmin})$, lands at a very different detector location compared to the other observations. However, this argument is not developed to the 


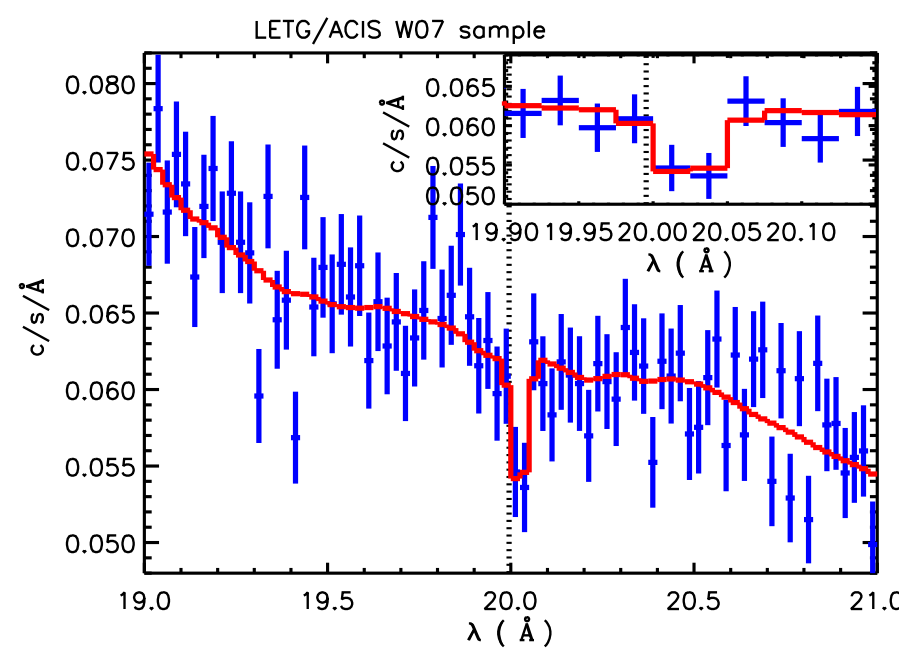

Fig. 6. Co-added LETG/ACIS data from Williams et al. (2007) observations (blue crosses), and the best-fit model (red line) consisting of a spline continuum and a redshifted $\mathrm{O}$ viII doublet Voigt profile with the redshift as a free parameter. The dotted vertical line indicates the expected wavelength of the $\mathrm{O}$ vIII centroid, that is, assuming the FUV redshift of $z=0.05405$.

point of comparing the results obtained with this observation with the rest of the sample.

\subsubsection{Methods}

We examined the above arguments by reanalysing the LETG/ACIS data used by Fang et al. (2007) and Williams et al. (2007). The data used in the two studies are almost the same, with the exception that the latter did not include the very high offset observation 3669 (42 ks), but did include an additional observation, 3668 (14 ks). Also, Williams et al. (2007) did not shift the energy scales of the two observations as Fang et al. (2007) did. Nevertheless, the two works yielded a significant detection of $\mathrm{O}$ VIII at $z \approx 0.054-0.055$ with consistent $\mathrm{EW}$ values.

We processed the data with the current public CIAO processing pipeline tool chandra_repro, and co-added the +1 and -1 order data using the tool combine_grating_spectra. We used a bin size of $25 \mathrm{~m} \AA$ as in Fang et al. (2007). The inclusion of the $2 \%$ systematic uncertainties (see Sect. 3) had no significant effect because the statistical uncertainties are larger due to shorter exposure and lower effective area compared to that of RGS.

As in the case of RGS and HRC (see Sect. 3.2) we examined here the wavelength-scale calibration accuracy of each LETG/ACIS co-added spectrum using Galactic lines. We then fitted the $19-21 \AA$ band continuum with a spline model ${ }^{2}$ with a grid step of $0.75 \AA$ to mimic the continuum modelling of Fang et al. (2007), where the polynomial fits effectively removed features broader than $0.7 \AA$. We then added a redshifted O VIII slab model to investigate the tentative WHIM line.

\subsubsection{Tests with the Williams et al. (2007) sample}

The Galactic $\mathrm{O}_{\mathrm{I}}$ and $\mathrm{O}$ VII lines in our co-added Williams et al. (2007) sample spectrum were unambiguously detected and indicated that the wavelength scale is very accurate; a possible

\footnotetext{
2 In case of the $\mathrm{O} \mathrm{VIII}_{\mathrm{A} 3}$ line, the usage of the more complex continuum, including the Galactic absorbers as in Sect. 3.4, yielded a negligible effect on the $\mathrm{O}$ vIII equivalent width in the narrow band we used.
}

Table 4. Tests of LETG/ACIS data at $\lambda \sim 20 \AA$.

\begin{tabular}{lcc}
\hline \hline Sample & $\begin{array}{c}\lambda \\
\AA\end{array}$ & $\begin{array}{c}\text { EW } \\
\text { mA }\end{array}$ \\
\hline W07 $^{a}$ & $20.03 \pm 0.01$ & $8.2 \pm 2.3$ \\
$\mathrm{~W} 07+^{b}$ & $20.03 \pm 0.01$ & $6.5 \pm 2.7$ \\
${\mathrm{~W} 07-{ }^{c}}^{c}$ & $20.02 \pm 0.01$ & $13.4 \pm 5.0$ \\
$3669^{d}$ & $20.01_{-0.02}^{+0.01 g}$ & $6.9 \pm 3.2$ \\
Final $^{e}$ & $20.02 \pm 0.01$ & $6.0 \pm 1.9$ \\
Final and 3669 $^{f}$ & $20.02 \pm 0.01$ & $6.2 \pm 1.7$ \\
\hline
\end{tabular}

Notes. ${ }^{(a)}$ Observations used in Williams et al. (2007), positive and negative first orders co-added. ${ }^{(b)}$ Observations used in Williams et al. (2007), but only positive first order. ${ }^{(c)}$ Observations used in Williams et al. (2007), but only negative first order. ${ }^{(d)}$ Large pointing offset observation 3669. ${ }^{\left({ }^{e}\right)}$ Using all the public data in Oct 2017 obtained with $\Delta y=+1.5$ arcmin and SIM $-Z=-8 \mathrm{~mm}$. ${ }^{(f)}$ Combining the results of the final sample and of the observation 3669. ${ }^{(g)}$ After correcting the wavelength scale as indicated by the shift of the wavelength of the Galactic O I line.

shift is smaller than the statistical uncertainties of $\pm 6 \mathrm{~m} \AA$, similarly to in Williams et al. (2007). The WHIM O vIII modelling of the 19-21 $\AA$ band data yielded a significant $(3.6 \sigma)$ detection of a line at $\lambda=20.03 \pm 0.01$, identical to Williams et al. (2007; see Fig. 6 and Table 4). Our EW $=8.2 \pm 2.3 \mathrm{~m} \AA$ is consistent with Williams et al. $(2007$; EW $=7.5 \pm 2.1 \mathrm{m \AA})$.

We then examined, whether the above detection may be an artifact produced by the node boundary (see above). We utilised the argument that the tentative $20 \AA$ line in the positive and negative first-order spectrum lands at a very different region of the detector. Thus, consistency of the wavelengths and equivalent widths of the lines in both orders would indicate a true astrophysical origin for the line. We used the same sample as (Williams et al. 2007), but this time separately for the positive and negative first-order co-added spectra.

In the case of the positive order, the Galactic lines did not allow a significant wavelength scale shift. Around $20 \AA$ the data indicated a line-like feature at $\lambda=20.03 \pm 0.01$, that is, identical to that found when using the co-added positive and negative firstorder spectra above. The equivalent width measurement of $6.5 \pm$ $2.7 \mathrm{~m} \AA$ yields a detection significance of $2.4 \sigma$ (see Fig. 7 and Table 4).

In the case of the negative order, no Galactic line was unambiguously detected. At the vicinity of $20 \AA$ we detected an absorption line at $\lambda=20.02 \pm 0.01 \AA$, consistent with the positive order (see Fig. 8 and Table 4). We measured EW = 13.4 $\pm 5.0 \mathrm{~m} \AA$ for this line, $(2.8 \sigma)$, consistent with the positive order.

While the statistical significances of the positive- and negative-order lines are not very high individually, their centroids are consistent within $1-3 \sigma$ with that of O vIII Ly $\alpha$ $(20.00 \AA$ ) at $z=0.05425$, the redshift of FUV absorber A3 (see Table 1). This match improves the probability of the line feature being a true astrophysical signal (see below).

\subsubsection{Tests with the large offset observation 3669}

We then considered the Fang et al. (2007) argument that the $20 \AA$ line in the large off-axis observation 3669 lands at a different detector region compared to the above lower offset sample case, and therefore is not affected by the same node boundary. 
Table 5. Final sample of LETG/ACIS observations.

\begin{tabular}{cc}
\hline \hline OBS. ID. & Exposure (ks) \\
\hline 2335 & 29 \\
3168 & 29 \\
3668 & 13 \\
3707 & 27 \\
4416 & 46 \\
6090 & 28 \\
6091 & 29 \\
6874 & 28 \\
6924 & 9 \\
6927 & 27 \\
7293 & 9 \\
8388 & 29 \\
10662 & 28 \\
\hline
\end{tabular}

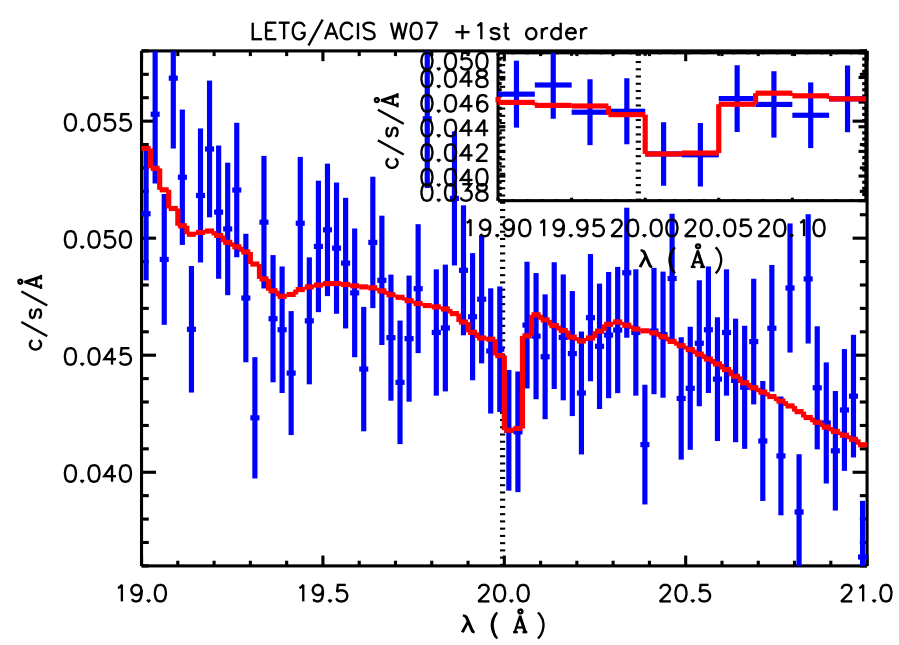

Fig. 7. As in Fig. 6 but using only positive first-order data.

Therefore it is very unlikely that random fluctuations would yield consistent lines using observation 3669 and the Williams et al. (2007) sample above.

The centroid of the Galactic O I (O viI was not useful) absorption line in observation 3669 was shifted towards lower wavelengths by $40 \pm 10 \mathrm{~m} \AA$. Such a large wavelength shift may be due to complications of the calibration of large offset observations.

At wavelengths around $20 \AA$ we found a $2.2 \sigma$ indication for an absorption line at $\lambda=19.97_{-0.02}^{+0.01} \AA$ (see Fig. 9). Since 20$\AA$ photons land quite far from the centre of the field of view (FOV) in such a high offset observation, the energy resolution is degraded. This is seen via the broad, $\sim 75-\mathrm{m} \AA$-wide feature in the data (see Fig. 9). Due to the different amount of broadening at different offset angles, it is important not to co-add observations with different offsets. The energy redistribution function shows a similarly broad feature, indicating that the off-set line spread function is well calibrated. Using the slab model we measured EW $=6.9 \pm 3.2 \mathrm{~m} \AA$ for this line (no additional broadening required) which is consistent with our measurement using the Williams et al. (2007) sample; see Sect. 4.4.3. Shifting the line centroid by $+40 \pm 10 \mathrm{m \AA}$, as indicated by the Galactic OI, the corrected wavelength $\lambda=20.01_{-0.02}^{+0.01} \AA$ of the O vIII line is consistent with that derived above using the Williams et al. (2007) sample (see Fig. 9 and Table 4).

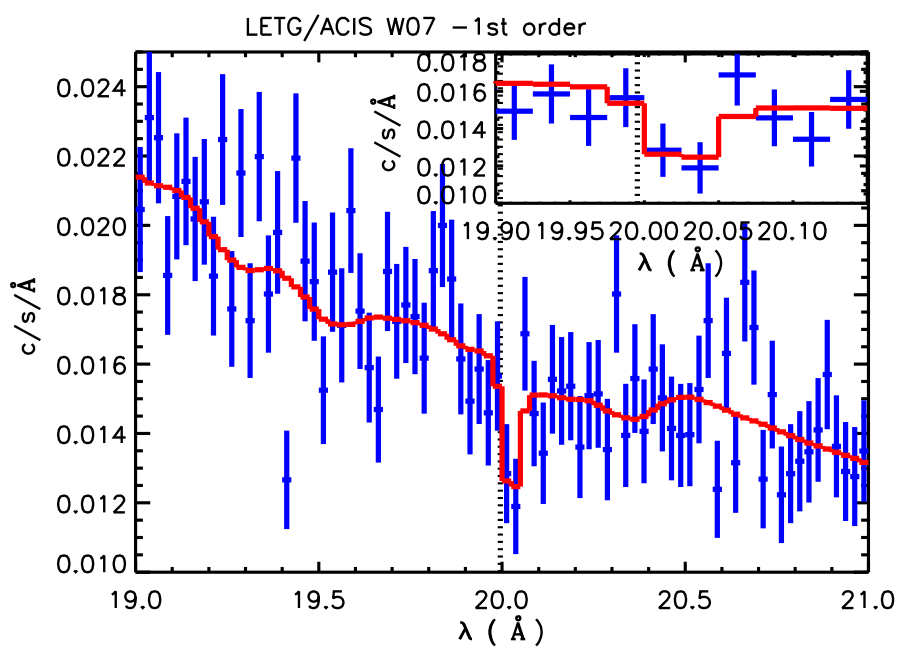

Fig. 8. As in Fig. 6 but using only negative first-order data.

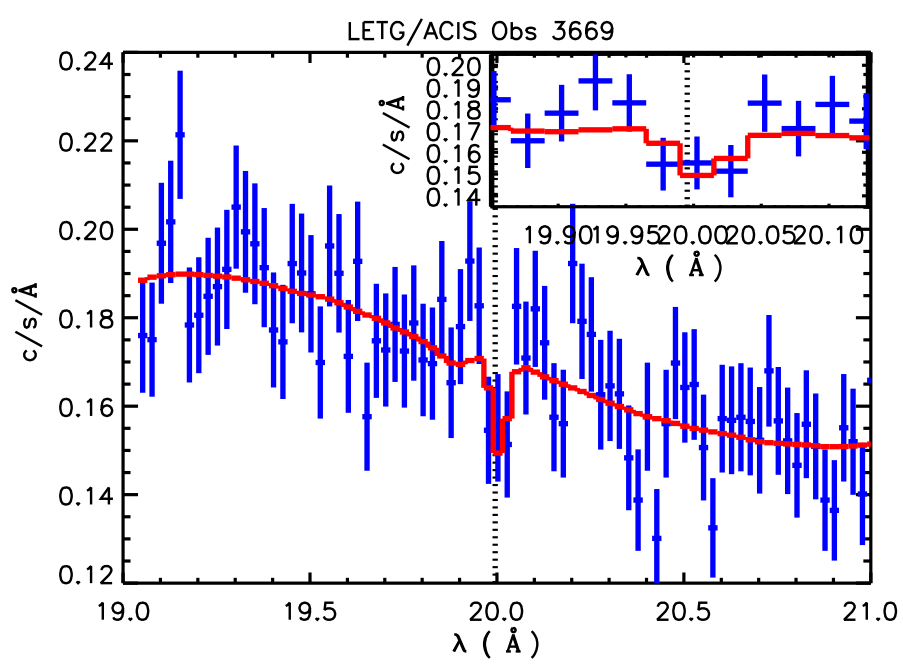

Fig. 9. As in Fig. 6 but using only observation 3669. The wavelengths of the data and the best-fit model are shifted by $+40 \mathrm{~m} \AA$, as suggested by the Galactic $\mathrm{O}_{\mathrm{I}}$ and $\mathrm{O}$ VII lines.

While the statistical significance of the line detection is relatively low, the agreement in wavelength with that of O vIII Ly $\alpha$ at the redshift of the FUV absorber A3 is in support of an astrophysical origin for this line.

\subsubsection{The final sample}

We then considered the additional useful observations of PKS 2155-304 obtained after the works published by Williams et al. (2007) and Fang et al. (2007), available in October 2017 (Table 5). Due to the indicated problems with the large offset pointing wavelength scale calibration (see Sect. 4.4.4) we selected only the observations obtained with the same offset setting as in the above works, that is, $\Delta y=+1.5 \operatorname{arcmin}$ and SIM $-Z=-8 \mathrm{~mm}$. Our co-added spectrum has an exposure time of $330 \mathrm{ks}$. The study of the wavelengths of the Galactic $\mathrm{O}_{\mathrm{I}}$ and $\mathrm{O}$ vII lines allowed no wavelength scale shift. We significantly detected a line at $z=0.0554 \pm 0.0004$ or $\lambda=20.02 \pm 0.01 \AA$ (see Fig. 10 and Table 4) with $\mathrm{EW}=6.0 \pm 1.9 \mathrm{~mA}(3.2 \sigma)$, consistent with our analysis of observation 3669 (see Sect. 4.4.4).

We did not co-add the data from the observation 3669 to that of the final sample due to the indicated significant wavelength 


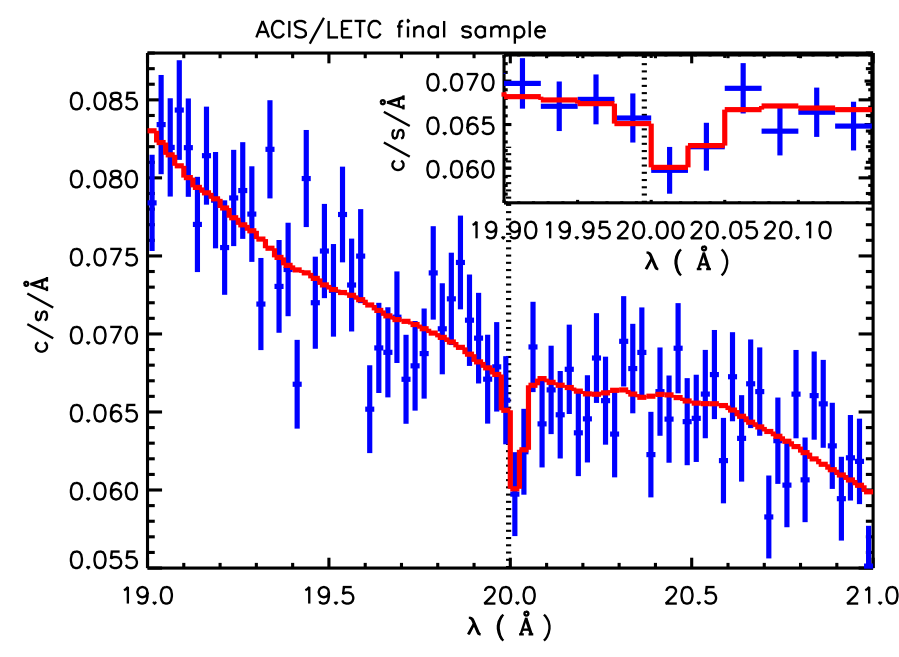

Fig. 10. As Fig. 6 but using the "final" sample.

shift. Since PKS 2155-304 was very bright during observation 3669 , it has significant statistical weight compared to the final sample. We thus combined the measurements of the equivalent width using the spectra of the final sample and the observation 3669 into an error-weighted average, obtaining $\mathrm{EW}=6.2 \pm 1.7$ for the $\lambda \approx 20 \AA$ line which is thus detected at quite a high statistical significance of $3.7 \sigma$.

\subsection{Summary}

Our measurements of the positive and negative orders of the Williams et al. (2007) sample and the large offset observation 3669 yielded consistent wavelengths and equivalent widths for the $\mathrm{O}$ VIII Ly $\alpha$ line, indicating that the systematic uncertainties are small. Our tests therefore indicate that the feature is very likely a true astrophysical signal. Assuming that the upper limit of the EW as given by RGS1 is also correct, we estimated the probability that the ACIS measurement is consistent with that of the RGS1. Applying the $\chi^{2}$ statistics to the ACIS analysis yielded a very low $(0.7 \%)$ probability for such a situation. The probability would become even smaller when considering the matches obtained with the above tests of positive and negative orders and the offset observation which cannot be derived analytically.

On the other hand, it is also very unlikely that $\sim 1.2 \mathrm{Ms}$ of RGS1 data and $\sim 300 \mathrm{ks}$ of HRC data could have missed a true O vIII Ly $\alpha$ line (see Sect. 4.3) with EW $=6 \mathrm{~m} \AA$, as measured with ACIS. Applying the $\chi^{2}$ statistics to the RGS1 analysis reveals a probability of $0.006 \%$ for such an incidence.

Complicated and extensive simulations would be needed to improve the accuracy of the above probabilities. However, we think that this is not useful since both probabilities, that is, the one for the ACIS detection being true and the one for the RGS1/HRC non-detection being true would be extremely high, and thus we could not conclude which case is significantly better. Given the very convincing $\mathrm{X}$-ray spectral evidence for and against the existence of the $\lambda \sim 20 \AA$ absorption line, we cannot make any conclusions as to whether or not the feature is a true astrophysical line.

\section{Discussion}

\subsection{The spatial co-incidence of O VII absorbers at the location of $\mathrm{O} v \mathrm{vl}$ absorbers}

In this work we assume the co-location of the warm and hot WHIM in order to enable the usage of the robustly detected warm WHIM in the FUV band as a tracer of the cosmologically interesting hot WHIM. We performed an X-ray follow-up of the O vil line in the PKS 2155-304 sight line at the two redshifts where the previous FUV observations (Tilton et al. 2012) yielded $\mathrm{O}$ vi detections at the level of $\left.\log N\left(\mathrm{O} \mathrm{vI}_{(\mathrm{cm}}{ }^{-2}\right)\right) \sim 14$. We obtained no significant detection and upper (RGS1) limits of $\log N\left(\mathrm{O}\right.$ VII $\left.\left(\mathrm{cm}^{-2}\right)\right) \leq 14.5$ and $15.2^{3}$ for the two absorbers, that is, A3 and A4 (see Table 2 and Fig. 1).

Considering the simulations of Cen (2012), the basic problem in our work is the short length of the studied redshift path, $\mathrm{d} z=0.1$. The above simulations indicated approximately one $\mathrm{O}$ vi absorber with $\log N\left(\mathrm{O}\right.$ vI $\left.\left(\mathrm{cm}^{-2}\right)\right) \sim 14$ and one $\mathrm{O}$ vII absorber at the level of $\log N\left(\mathrm{O} \mathrm{vII}\left(\mathrm{cm}^{-2}\right)\right) \sim 15$ within our path length, on average. The observational values for the above absorbers are two (O vi) and zero (O vII), similar to the prediction. Additionally, in the above simulation (Cen 2012), the probability of finding an absorber with $\log N\left(\mathrm{O}\right.$ VII $\left.\left(\mathrm{cm}^{-2}\right)\right) \sim 15$ at the location of an absorber with $\log N\left(\mathrm{O} \mathrm{vI}\left(\mathrm{cm}^{-2}\right)\right) \sim 14$ is less than $10 \%$. Therefore, we should increase the studied path length at least by an order of magnitude to get a meaningful sample for comparisons with the simulations. As we demonstrated in this work, the study of the $\mathrm{O}_{\mathrm{vI}}$ line at even a small path length at the level $\log N\left(\mathrm{O}\right.$ vII $\left.\left(\mathrm{cm}^{-2}\right)\right) \sim 15$ is very challenging. The requirements for necessary signal-to-noise ratios with the current instruments translate into very long exposures of several hundred kiloseconds. We plan to systematically probe the current archival highresolution X-ray spectra at the locations of the FUV-detected warm WHIM in a future study.

\section{2. $L E T C / A C I S \lambda \sim 20 \AA$ feature}

The LETG/ACIS feature at $\lambda \sim 20 \AA$ in our final sample (see Fig. 10), if interpreted as being due to $\mathrm{O}$ VIII at absorber A3 $(z \sim 0.054)$, corresponds to $\log N\left(\mathrm{O} \operatorname{vIII}\left(\mathrm{cm}^{-2}\right)\right)=15.7_{-0.2}^{+0.1}$. At consistent redshifts there is very securely detected FUV absorption (see Table 1) yielding $\log N\left(\mathrm{O}\right.$ vi $\left.\left(\mathrm{cm}^{-2}\right)\right)=13.6 \pm 0.1$ (Tilton et al. 2012). The simulations of Cen (2012) indicate that in such a small redshift path as studied in our work $(\mathrm{d} z \sim 0.1)$ there should be on average only $\sim 0.1 \mathrm{O}$ vII absorbers with column density exceeding $10^{15.7} \mathrm{~cm}^{-2}$. Since the IllustrisTNG simulations (Nelson et al. 2018) indicate that clustering of $\mathrm{O}$ VIII ions on megaparsec scales is an order of magnitude smaller than that of $\mathrm{O}$ VII ions, the probability for finding $\mathrm{O}$ VIII absorbers is much smaller than that $(\lesssim 10 \%)$ for O vII discussed above in Sect. 5.1. Therefore, the probability of the LETG/ACIS $\lambda \sim 20 \AA$ feature being due to the astrophysical $\mathrm{O}$ VIII absorber co-located with the FUV-detected $\mathrm{O}$ vi absorber is at the very low level of $\lesssim 0.1 \%$.

Assuming that the LETG/ACIS $\lambda \sim 20 \AA$ feature is due to $\mathrm{O}$ vIII, the absence of significant $\mathrm{O}$ VII absorption at $\mathrm{A} 3$ indicates two distinct gas phases: the FUV-detected warm phase with $\log T(\mathrm{~K}) \lesssim 6$ and the X-ray-detected hot phase with $\log T(\mathrm{~K}) \gtrsim$ 6. Such a configuration is possible in the WHIM embedded in a large-scale filament. The cosmological simulations EAGLE (Schaye et al. 2015) and IllustrisTNG (Nelson et al. 2018), for example, suggest that the hottest WHIM is concentrated along filament axes while the warm WHIM occupies much larger surrounding volumes. Therefore, most of the random sight lines are expected to contain only the warm FUV WHIM, consistent with the simulations of Cen (2012). We suggest that in a few lucky incidences (as may be the case for A3, as well as for the

3 The value is higher due to complications with the overlapping Galactic O Iv line. 
Sculptor Wall, Buote et al. 2009; Fang et al. 2010 and 3C273, Ahoranta et al., in prep.) when the sight line passes very close to the filament axis, both warm and hot WHIM may be co-located and therefore the FUV and X-ray spectra would exhibit WHIM absorption lines at similar redshifts.

Assuming that a) the LETG/ACIS $\lambda \sim 20 \AA$ feature is due to $\mathrm{O}$ VIII embedded in the WHIM in an intervening large scale filament, b) the metal abundances in the $\mathrm{A} 3$ absorber are 0.1 times solar, c) the temperature of the WHIM is quite high $10^{6.5} \mathrm{~K}^{4}$ to keep the $\mathrm{O}$ VII column density below the detection limit of $10^{15} \mathrm{~cm}^{-2}$, and d) that the system is in collisional ionisation equilibrium, the LETG/ACIS measurement $\log N\left(\mathrm{O}_{\text {VIII }}\left(\mathrm{cm}^{-2}\right)\right)=$ $15.7_{-0.2}^{+0.1}$ corresponds to an equivalent hydrogen column density level of $10^{20} \mathrm{~cm}^{-3}$. Such a hot model also contains lines from $\mathrm{Ne}$ IX (rest frame $\lambda \sim 13.447 \AA$ ) and Fe XVII (rest frame $\lambda \sim 15.014 \AA$ ). The Ne Ix line is consistent with the ACIS data. The FexvII line is slightly overpredicted with such a model, but within the statistical uncertainties the iron abundance is consistent with 0.1 times solar used for oxygen. Therefore, the CIE modelling did not rule out the $\mathrm{O}$ viII line.

Adopting the typical baryon overdensity range of 10-100 for the WHIM implies that the path through the WHIM should be in excess of $10 \mathrm{Mpc}$. This in turn requires a co-incidence of a major filament being oriented very closely along the sight line. We plan to address the puzzle of the $\mathrm{A} 3 \mathrm{O}$ vIII line in a future work by utilising the galaxy distribution around the possible X-ray absorber in order to detect or rule out a major galactic filament crossing the PKS 2155-304 sight line at the matching redshift.

\subsection{Transient LETG/ACIS $\lambda \sim 20 \AA$ absorption?}

In the case of the blazar H2356-309, Fang et al. (2011) detected a transient $\mathrm{O}$ VIII absorption feature (duration $\approx 100 \mathrm{ks}$ ) at the surface of the blazar using LETG/HRC. The feature was found to be consistent with thermal instability of the absorber and with an outflow of absorbing material. We examine here whether a similar scenario could explain the LETG/ACIS detection of the line feature at $\lambda \sim 20 \AA$ in the case of PKS 2155-304.

LETC/ACIS observation 3669 (not included in the Williams et al. 2007 sample; see Sect. 4.4.4) and the Williams et al. (2007) sample (Sect. 4.4.3) both show the line consistently. This can be interpreted as two distinct transients with similar strength. It is not very likely that one or two transient absorption events took place when LETG/ACIS was observing PKS 2155-304, and none took place when RGS or LETG/HRC observed PKS 2155-304. Yet the sampling of PKS 2155-304 with RGS and LETG is not frequent enough to completely rule out this possibility.

If the X-ray feature at $\lambda \sim 20 \AA$, measured with LETG/ACIS only, is due to $\mathrm{O}$ VIII at the blazar surface $(z \approx 0.12)$, the outflow velocity is $\mathrm{d} z \times c \approx(1.12-1.05) \times c \approx 20000 \mathrm{~km} \mathrm{~s}^{-1}$ towards us. Such ultra-fast outflows (UFOs) have been observed in the X-ray spectra of many Seyfert galaxies (e.g. Tombesi \& Cappi 2014). However, we are not aware of any detection of similarly fast outflow in a blazar such as PKS 2155-304.

Let us assume that blazars have not been sufficiently surveyed to robustly rule out the UFO scenario for PKS 2155-304; the LETG/ACIS X-ray feature is nonetheless too narrow and too weak compared to absorption lines measured in UFOs related to Seyferts (e.g. Tombesi \& Cappi 2014). Furthermore, in the UFO

\footnotetext{
4 If the temperature is higher, that is, less optimal for $\mathrm{O}$ vIII production, the hydrogen density and thus the path length would become larger.
}

scenario the outflow at the blazar surface happens to have such a velocity that its combined effect with the Hubble velocity redshifts the $\mathrm{O}$ VIII line to $\lambda \approx 20 \AA$, which coincides with the wavelength of $\mathrm{O}$ VIII if originating from an absorber with only Hubble velocity at the location of the FUV absorber A3 ( $\mathrm{O}$ vI and two BLAs; see Table1).

In summary, given the above problems, it is very unlikely that the transient absorption explains the LETG/ACIS $\lambda \sim 20 \AA$ feature.

\section{Conclusions}

We analysed all available useful high-resolution X-ray spectral data in the direction of the blazar PKS 2155-304 at the redshifts where FUV observations (Tilton et al. 2012) have obtained indications of the warm WHIM. The FUV measurements consist of two absorbers with $\log N\left(\mathrm{O} v \mathrm{vi}\left(\mathrm{cm}^{-2}\right)\right)=13.6 \pm 0.1$ : one with $\log N\left(\operatorname{Si~} \mathrm{IV}\left(\mathrm{cm}^{-2}\right)\right) \sim 12.1 \pm 0.1$, and several BLAs (H I broadened by $\left.b \geq 40 \mathrm{~km} \mathrm{~s}^{-1}\right)$ at the level of $\log N\left(\mathrm{H} \mathrm{I}\left(\mathrm{cm}^{-2}\right)\right) \sim 12-14$ (see Table 1). The studied redshift path is $\mathrm{d} z \sim 0.1$. The analysis yielded the following conclusions:

- We did not obtain any significant detections of O vII He $\alpha$ absorption lines, the most likely hot WHIM tracer, at the five (considering the $\mathrm{O}$ VI lines and BLAs) or two (considering only the O vi lines) FUV-based X-ray follow-up redshifts. The non-detections correspond to upper RGS1 limits of $\log N\left(\mathrm{O}\right.$ viI $\left.\left(\mathrm{cm}^{-2}\right)\right) \leq 14.5-15.2$

- At five of the six studied redshifts we did not detect any significant $\mathrm{O}$ VIII $\operatorname{Ly} \alpha$ absorption line. The upper limit is $\log N\left(\mathrm{O}_{\text {VIII }}\left(\mathrm{cm}^{-2}\right)\right) \lesssim 15$.

- The LETG/ACIS combination yielded a significant $(3.7 \sigma)$ detection of an absorption-line-like feature at $\lambda \sim 20 \AA$. If interpreting this as a true absorption line due to $\mathrm{O}$ vIII, its redshift matches one of the six FUV-based X-ray follow-up redshifts $(z \sim 0.054)$.

- The data from RGS1 and LETG/HRC did not detect the LETG/ACIS $\lambda \sim 20 \AA$ feature. Given the high statistical quality of the RGS1 data, the possibility of RGS1 accidentally missing the true line at $\lambda 20 \AA$ is very low, $0.006 \%$.

- Considering the simulations (Cen 2012, Nelson et al. 2018), the probability of the LETG/ACIS $\lambda \sim 20 \AA$ feature being due to an astrophysical $\mathrm{O}$ vIII absorber co-located with the FUV-detected $\mathrm{O}$ vi absorber is at the very low level of $\lesssim 0.1 \%$.

- We cannot completely rule out the very unlikely possibility that the LETG/ACIS $20 \AA$ feature is due to a transient event located close to the blazar.

Acknowledgements. We acknowledge the support by the Estonian Research Council grants PUT246, IUT26-2, IUT40-2, and by the European Regional Development Fund (TK133 and MOBTP86). Thanks to the Chandra X-ray observatory HelpDesk. JN acknowledges the funds from a European Horizon 2020 program AHEAD (Integrated Activities in the High Energy Astrophysics Domain), and from FINCA (the Finnish Centre for Astronomy with ESO). Thanks to Jelle de Plaa for his help with the Spex analysis.

\section{References}

Branchini, E., Ursino, E., Corsi, A., et al. 2009, ApJ, 697, 328

Buote, D. A., Zappacosta, L., Fang, T., et al. 2009, ApJ, 695, 1351

Cagnoni, I., Nicastro, F., Maraschi, L., Treves, A., \& Tavecchio, F. 2004, ApJ, 603,449

Cen, R. 2012, ApJ, 753, 17

Cen, R., \& Ostriker, J. P. 1999, ApJ, 514, 1

Cui, W., Borgani, S., Dolag, K., Murante, G., \& Tornatore, L. 2012, MNRAS, 423, 2279 
Cui, W., Knebe, A., Yepes, G., et al. 2018, MNRAS, 473, 68

Danforth, C. W., \& Shull, J. M. 2008, ApJ, 679, 194

Danforth, C. W., Stocke, J. T., France, K., Begelman, M. C., \& Perlman, E. 2016, ApJ, 832, 76

Davé, R., Cen, R., Ostriker, J. P., et al. 2001, ApJ, 552, 473

de Vries, C. P., den Herder, J. W., Kaastra, J. S., et al. 2003, A\&A, 404, 959

de Vries, C. P., den Herder, J. W., Gabriel, C., et al. 2015, A\&A, 573, A128

Dolag, K., Meneghetti, M., Moscardini, L., Rasia, E., \& Bonaldi, A. 2006 , MNRAS, 370,656

Fang, T., Marshall, H. L., Lee, J. C., Davis, D. S., \& Canizares, C. R. 2002, ApJ, 572, L127

Fang, T., Canizares, C. R., \& Yao, Y. 2007, ApJ, 670, 992

Fang, T., Buote, D. A., Humphrey, P. J., et al. 2010, ApJ, 714, 1715

Fang, T., Buote, D. A., Humphrey, P. J., \& Canizares, C. R. 2011, ApJ, 731, 46

Kaastra, J. S., Mewe, R., \& Nieuwenhuijzen, H. 1996, in UV and X-ray Spectroscopy of Astrophysical and Laboratory Plasmas, eds. K. Yamashita, \& T. Watanabe, 411

Lehner, N., Savage, B. D., Richter, P., et al. 2007, ApJ, 658, 680

Lodders, K., \& Palme, H. 2009, Meteorit. Planet. Sci. Suppl., 72, 5154

Nelson, D., Kauffmann, G., Pillepich, A., et al. 2018, MNRAS, 477, 450
Nevalainen, J., Tempel, E., Liivamägi, L. J., et al. 2015, A\&A, 583, A142 Nevalainen, J., Wakker, B., Kaastra, J., et al. 2017, A\&A, 605, A47 Nicastro, F., Senatore, F., Gupta, A., et al. 2016, MNRAS, 458, L123 Nicastro, F., Kaastra, J., Krongold, Y., et al. 2018, Nature, 558, 406 Richter, P., Nuza, S. E., Fox, A. J., et al. 2017, A\&A, 607, A48 Savage, B. D., Kim, T.-S., Wakker, B. P., et al. 2014, ApJS, 212, 8 Schaye, J., Crain, R. A., Bower, R. G., et al. 2015, MNRAS, 446, 521 Sembach, K. R., Wakker, B. P., Savage, B. D., et al. 2003, ApJS, 146, 165 Shull, J. M., Penton, S. V., Stocke, J. T., et al. 1998, AJ, 116, 2094 Shull, J. M., Tumlinson, J., \& Giroux, M. L. 2003, ApJ, 594, L107 Shull, J. M., Smith, B. D., \& Danforth, C. W. 2012, ApJ, 759, 23 Stocke, J. T., Keeney, B. A., Danforth, C. W., et al. 2013, ApJ, 763, 148 Stocke, J. T., Keeney, B. A., Danforth, C. W., et al. 2014, ApJ, 791, 128 Tilton, E. M., Danforth, C. W., Shull, J. M., \& Ross, T. L. 2012, ApJ, 759, 112

Tombesi, F., \& Cappi, M. 2014, MNRAS, 443, L104

Wakker, B. P., Savage, B. D., Sembach, K. R., et al. 2003, ApJS, 146, 1 Williams, R. J., Mathur, S., Nicastro, F., \& Elvis, M. 2007, ApJ, 665, 247 Williams, R. J., Mulchaey, J. S., \& Kollmeier, J. A. 2013, ApJ, 762, L10 Yao, Y., Tripp, T. M., Wang, Q. D., et al. 2009, ApJ, 697, 1784 